Math. Model. Nat. Phenom.

Vol. 3, No. 4, 2008, pp. 114-130

\title{
Local Collapses in the Truscott-Brindley Model
}

\author{
I. Siekmann ${ }^{a 1}$ and H. Malchow ${ }^{a}$ \\ ${ }^{a}$ Institut für Umweltsystemforschung, Universität Osnabrück, 49076 Osnabrück, Germany
}

\begin{abstract}
Relaxation oscillations are limit cycles with two clearly different time scales. In this article the spatio-temporal dynamics of a standard prey-predator system in the parameter region of relaxation oscillation is investigated. Both prey and predator population are distributed irregularly at a relatively high average level between a maximal and a minimal value. However, the slowly developing complex pattern exhibits a feature of "inverse excitability": Both populations show collapses which occur erratically both in space and in time. The nature of these collapses is analysed statistically and it is shown that the model behaviour can be interpreted as a resolution of the paradox of enrichment.
\end{abstract}

Key words: excitability, slow-fast cycles, spatiotemporal patterns, relaxation oscillations, paradox of enrichment

AMS subject classification: 35K57, 35Q80, 60H15

\section{Introduction}

The term "relaxation oscillations" was initially introduced by van der Pol [23] who investigated an electrical circuit. The mathematical theory of relaxation oscillations was mainly developed in the Russian literature, starting with a short note by Mishchenko and Pontryagin [13] and further elaborated in $[12,15]$. In general, from the mathematical point of view, periodic solutions of a singularly perturbed system of ordinary differential equations

$$
\begin{aligned}
\frac{d x}{d t} & =f(x, y), \\
\epsilon \frac{d y}{d t} & =g(x, y), \quad x \in \mathbb{R}^{m}, y \in \mathbb{R}^{n},
\end{aligned}
$$

\footnotetext{
${ }^{1}$ Corresponding author. E-mail: ivo.siekmann@uos.de
} 
are denoted relaxation oscillations, see the monograph [6] on relaxation oscillations in ecological models (which is, however, focussed on delay ODE models).

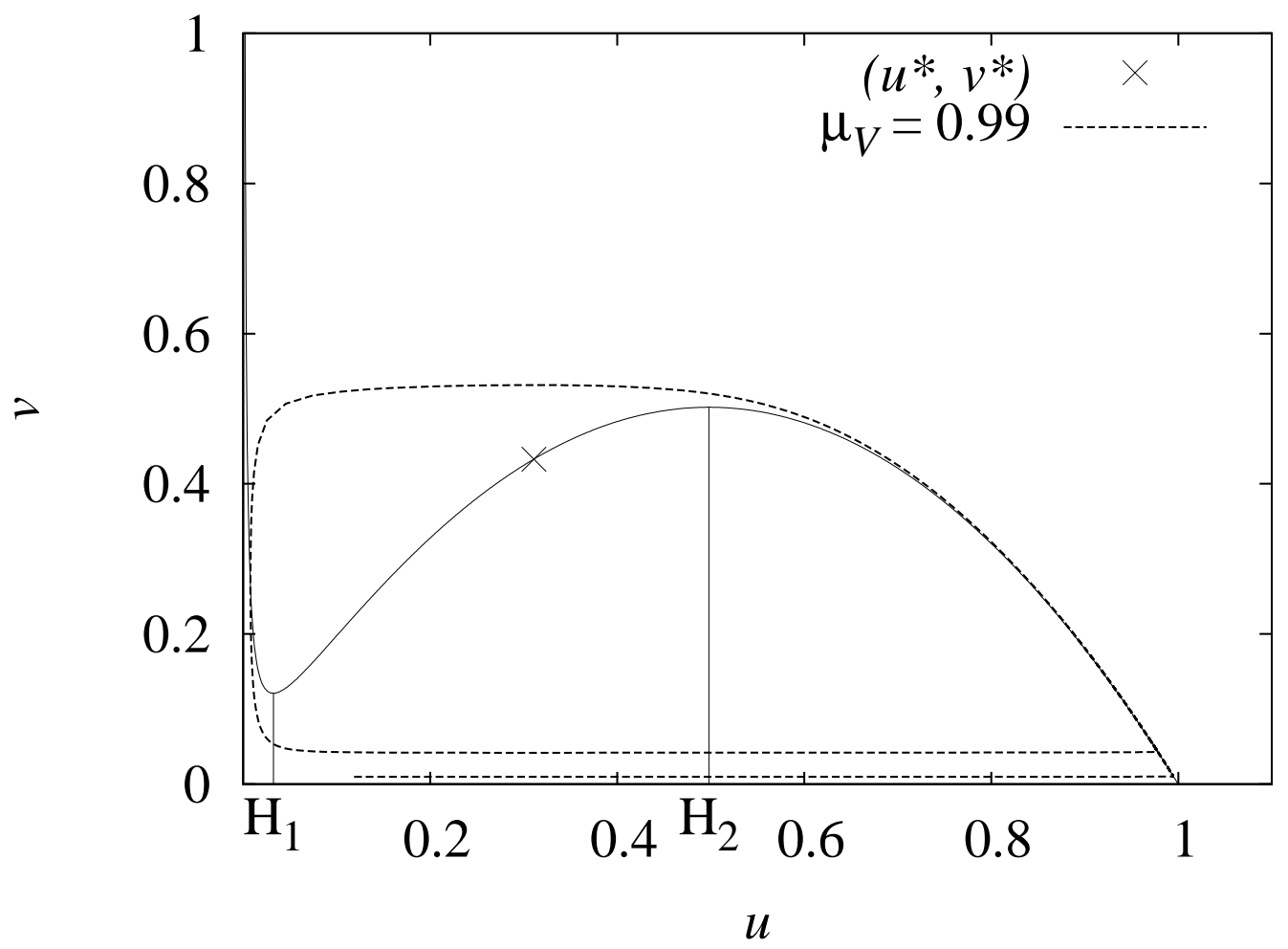

Figure 1: Phase space diagram of the Holling type III prey-predator model: The prey nullcline is plotted with solid lines. The two Hopf bifurcations are labelled ' $H_{1}$ ' and ' $H_{2}$ '. One limit cycle solution for the parameter set (3.1) is plotted with dashed lines, the corresponding stationary solution is indicated by a cross and labelled ' $\left(u^{*}, v^{*}\right)$ '.

Prey-predator models with Holling type III functional response have often been used to demonstrate cyclic collapses, cf. the classical paper by Ludwig et al. [10]. In the parameter range of relaxation oscillations, the behaviour is characterised by two clearly seperated time scales: For parameter sets close to the Hopf bifurcation $\mathrm{H}_{2}$, see the phase space diagram with the prey nullcline shown in Figure 1, the populations remain at nearly the same level for a long time but suddenly collapse as soon as a certain threshold, say for the predator population, denoted $v_{\max }$, is reached, see Figure 2.

Opposed to excitable solutions where a stable stationary state is reached after a single spike, relaxation oscillations consist of a train of spikes. In the phase space diagram (Figure 1) this behaviour can be interpreted by the alternating movement on a "fast" and a "slow" manifold: In our case the prey nullcline is the slow manifold whereas the movement on lines which are nearly parallel to the $u$ axis is fast. Concerning the analysis of slow-fast systems, see [16]. In this article it will 


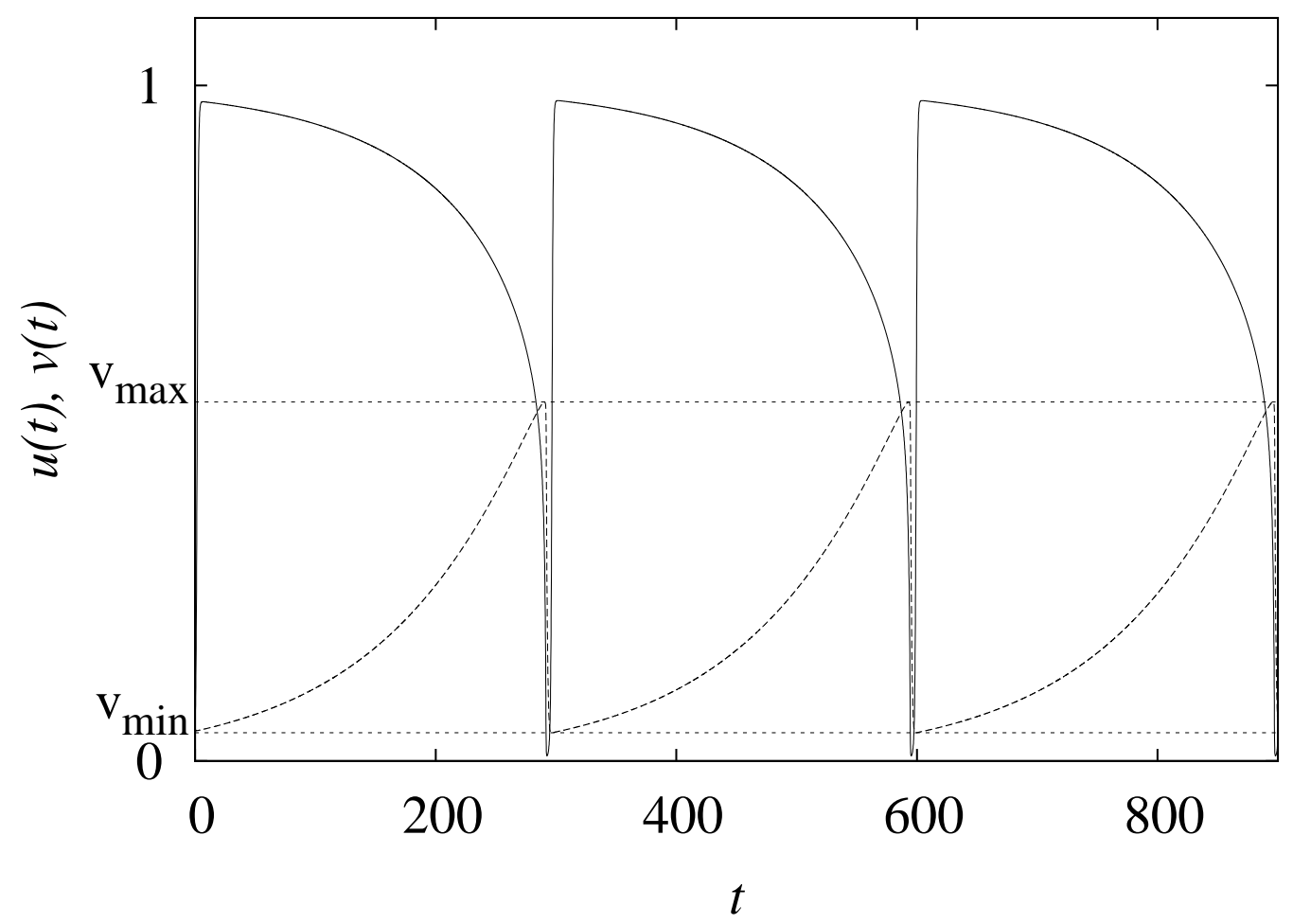

Figure 2: In order to identify critical values of the prey-predator dynamics, the local model is simulated for the choice of parameters given in Equation (3.1). Prey levels are plotted with solid lines, predator levels with dashed lines. The dotted lines show critical levels for predator densities. At a certain critical value $v_{\max }$ of the predator density, the prey population collapses whereas it explodes for a low predator density $v_{\text {min }}$.

be shown that slow-fast dynamics can lead to sudden local collapses whereas in most of the spatial area both populations are at high levels: If the predator level reaches $v_{\max }$ at a certain point, the prey and soon also the predator population collapse as to be expected from the local model. However, the spatial extension of these collapses is very limited; after a collapse, the prey population regrows explosively to its carrying capacity and the predator recovers slowly. This observation is interesting in the context of the famous "paradox of enrichment": Rosenzweig [17] observed that in a broad class of prey predator models an increase of the carrying capacity of the prey population leads to destabilisation of the interior stationary solution, i.e. the equilibrium which corresponds to coexistence of prey and predator. Instead both populations oscillate and if the carrying capacity is increased further, the oscillations become so sharp that the minimum values of both populations are close to extinction. This is a very counter-intuitive observation because it suggests that "enrichment", i.e. an improvement of the living conditions in the ecosystem might actually lead to extinction. Petrovskii et al. [14]—-see also [11, chapter 11.3.2] which provides a valuable source of references related to the paradox of enrichment-suggest that spatio-temporal chaos might pro- 
vide a mechanism which prevents from sharp oscillations in an enriched system. The results, which will be presented in the following, complement [14]: As a more general mechanism we suggest that slow-fast dynamics combined with spatial extension of the system are sufficient that both populations remain on a relatively high level.

\section{Spatio-temporal stochastic Truscott-Brindley model}

The Truscott-Brindley model [22], extended by diffusive movement of the species and stochastic variations driven by multiplicative noise, is investigated:

$$
\begin{aligned}
& \frac{\partial U}{\partial \tau}=r U\left(1-\frac{U}{K}\right)-F_{\max } \frac{U^{2}}{H^{2}+U^{2}} V+D \Delta U+\omega \zeta_{U} U, \\
& \frac{\partial V}{\partial \tau}=e F_{\max } \frac{U^{2}}{H^{2}+U^{2}} V-m_{V} V+D \Delta V+\omega \zeta_{V} V .
\end{aligned}
$$

$r$ denotes the growth rate of the prey, $K$ is the carrying capacity, $F_{\text {max }}$ is the maximal catch rate of the predator, $H$ is the half saturation constant of the Holling type III functional response, see [5]. $e$ is a so called efficiency factor which accounts for the fact that the predator is not capable of transforming each caught prey into the same amount of own biomass, $m_{V}$ is the natural mortality of the predator. Both species spread by diffusive movement with the same diffusion coefficient $D . \zeta_{U}$ and $\zeta_{V}$ stand for Gaussian noise with zero mean and unity variance. The factor $\omega$ describes the strength of the density-dependent noise which is used as a simple model for stochastic environmental variability.

By passing over to dimensionless variables

$$
u:=\frac{U}{K}, v:=\frac{V}{e K}, t:=e F_{\max } \tau
$$

and dimensionless parameters

$$
\rho:=\frac{r}{e F_{\max }}, h:=\frac{H}{K}, \mu_{V}:=\frac{m_{V}}{e F_{\max }}, d:=\frac{D}{e F_{\max } L^{k}}
$$

where the diffusion coefficient $D$ is scaled by the system length $L$ to the power $k=1,2$ of spatial dimensions, the transformed system reads

$$
\begin{aligned}
& \frac{\partial u}{\partial t}=\rho u(1-u)-\frac{u^{2}}{h^{2}+u^{2}} v+d \Delta u+\omega \zeta_{U} u, \\
& \frac{\partial v}{\partial t}=\frac{u^{2}}{h^{2}+u^{2}} v-\mu_{V} v+d \Delta v+\omega \zeta_{V} v .
\end{aligned}
$$




\section{Spatio-temporal dynamics}

A range of parameters is chosen where the dynamics of the local model is characterised by relaxation oscillations. Relaxation oscillations occur if the dynamics of the predator is much slower than the prey dynamics. Suitable choices of parameters are low values of $h$ and $\mu_{V}$ close to 1 . Biologically, this can be interpreted in the sense that the environment is "enriched" (because the carrying capacity $K$ is large compared to the half saturation constant $H$ of the predator) and that the mortality of the predator is close to but still below its growth rate $e F_{\max }$.

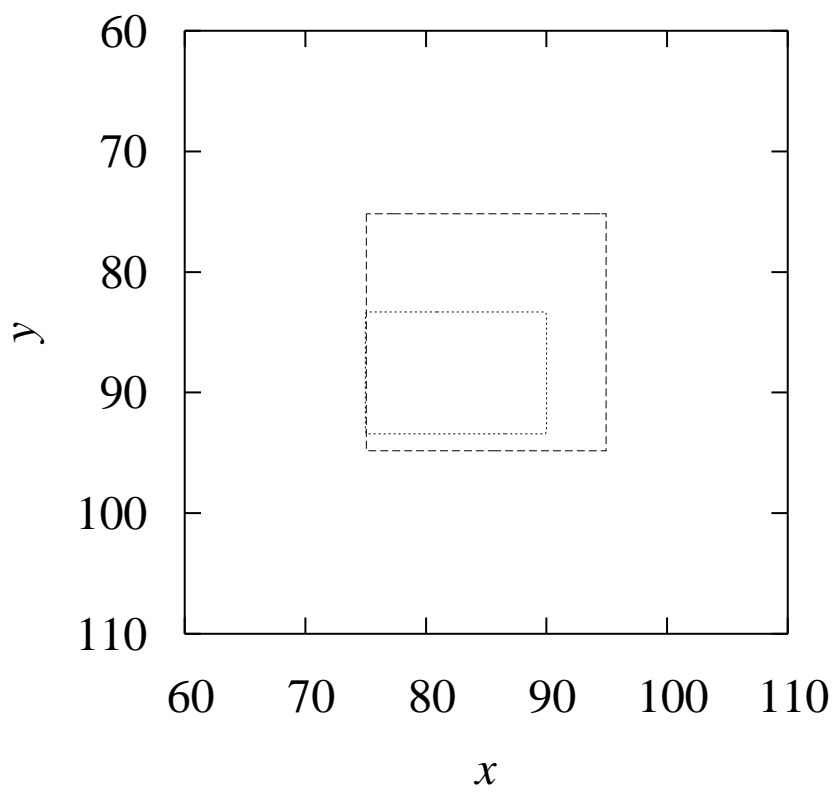

Figure 3: Initial conditions for the $500 \times 500$ grid with spatial step size $h_{x}=0.333$. The initial prey patch is bounded by a dashed, the initial patch of the predator by a dotted line.

The first choice of parameters which was used for simulation is

$$
\rho=2.0, \mu_{V}=0.99, h=0.03125, d=0.05 .
$$

The simulation was carried out on a $500 \times 500$ grid, each grid cell having a length of $h_{x}=0.333$ spatial units. For all simulations, a square of prey with the lower left corner $(75,75)$ and side length 20 was initialised with the value $u_{0}=0.1$ and covered by a rectangular patch with the lower left corner $(75,83)$ and side lengths 15 and 10 respectively was initialised with $v_{0}=0.2$. The rest of the space was set to 0 for both populations, see Figure 3 (it shall be noted that neither varying the initial conditions, nor choosing slightly different diffusion coefficients, nor slightly varying the other parameters leads to qualitative changes in the dynamics). The results are shown in Figure 4.

A complex pattern is formed very slowly in three stages: First, the prey grows quickly to its carrying capacity and occupies the whole space. At the same time (during the first few hundred 


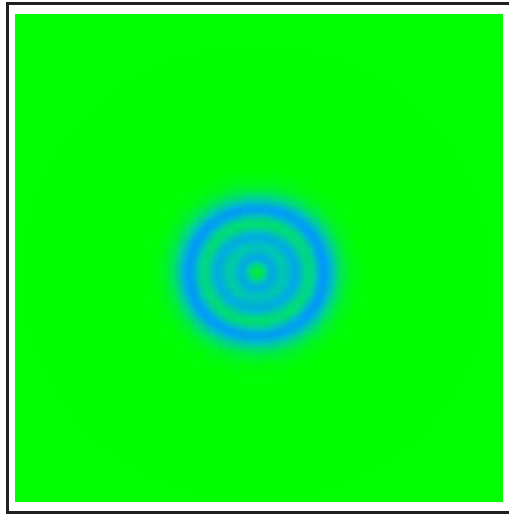

(a) $\mathrm{t}=700$

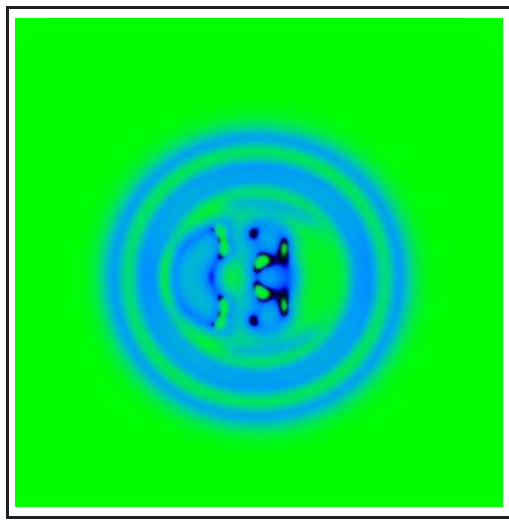

(d) $\mathrm{t}=1350$

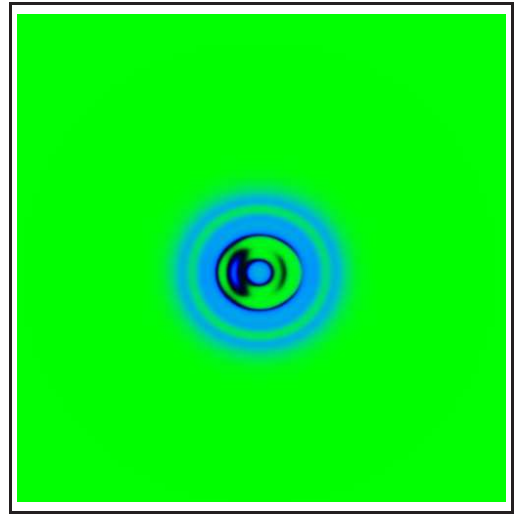

(b) $t=750$

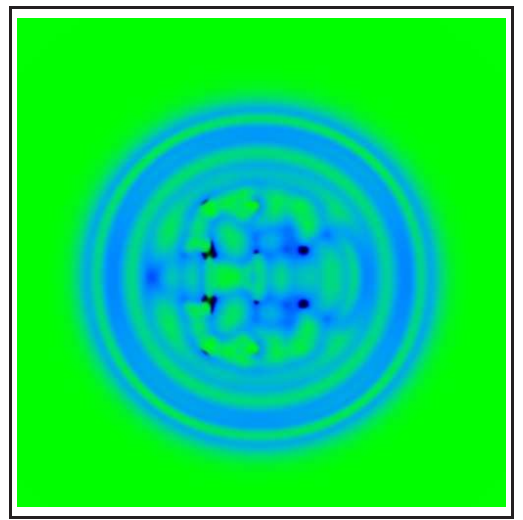

(e) $t=1575$

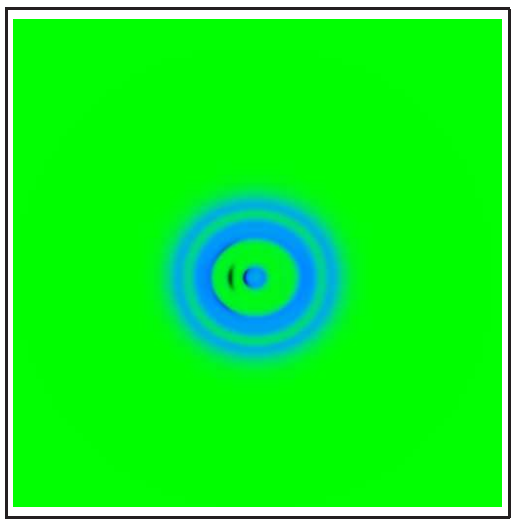

(c) $t=755$

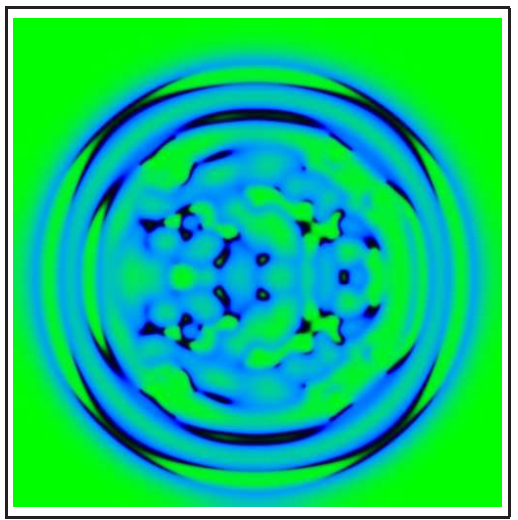

(f) $\mathrm{t}=1975$

Figure 4: Simulation of (2.1) for the choice of parameters (3.1) on a $500 \times 500$ grid; initial condition shown in Figure 3. The prey population is plotted green, the predator is plotted blue. Black areas indicate that in these regions in space both populations are close to extinction. In the beginning what looks like a target pattern (see (a)) already exhibits local collapses (see (b)), followed by explosive regrowth of the prey population (see (c)). Inside of what first looked like a target pattern, the collapses form complex structures (see (d),(e)); finally, black spots indicating collapses move along a "cellular" structure (see (f)).

time steps) the predator hardly seems to change at all. In the next phase the pattern resembles the well-known target pattern (see Figure 4a). However, already at this stage the pattern exhibits some kind of "inverse excitability": Occasionally, light blue rings and black spots appear which indicate very low levels of the prey population (see Figure 4b). At the same areas, also the predator population collapses nearly instantaneously. We denote this behaviour "inverse excitability" because most of the time the populations are at a resting state which is characterised by high levels where small perturbations might induce local collapses; however, excitability is usually defined as the possibility to "excite" a low-valued resting state. The dynamics results from the periodic collapses which could be observed in the local model: When the predator level has reached a critical value $v_{\text {max }}$ the prey population suddenly breaks down, see Figure 2 . As to be expected from the local 
dynamics, the life time of the black areas is not very long because each collapse is followed immediately by explosive regrowth of the prey population (see Figure 4c). Finally, at the last stage, the inside of what first looked like a target pattern becomes more and more irregular: Light greeen "cells" (where the prey population is relatively high, close to its carrying capacity) are separated by light blue "membranes" (where the prey is clearly below its carrying capacity). These "cells" do not change shape over a long time whereas black spots which mark low prey levels move quickly along the "membranes" showing a high variability both in shape and size (see Figures 4d-4f).

In general, it shows that the prey population is at a high level in most of the spatial area. To get a more quantitative idea of this observation we repeat the simulation with the parameter set (3.1) in one-dimensional space (see Figure 5).

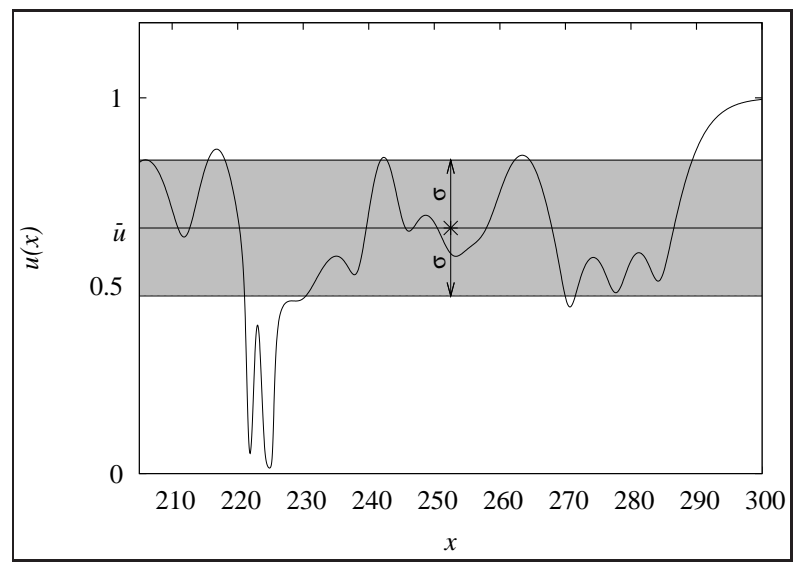

(a) $\mathrm{t}=2315$

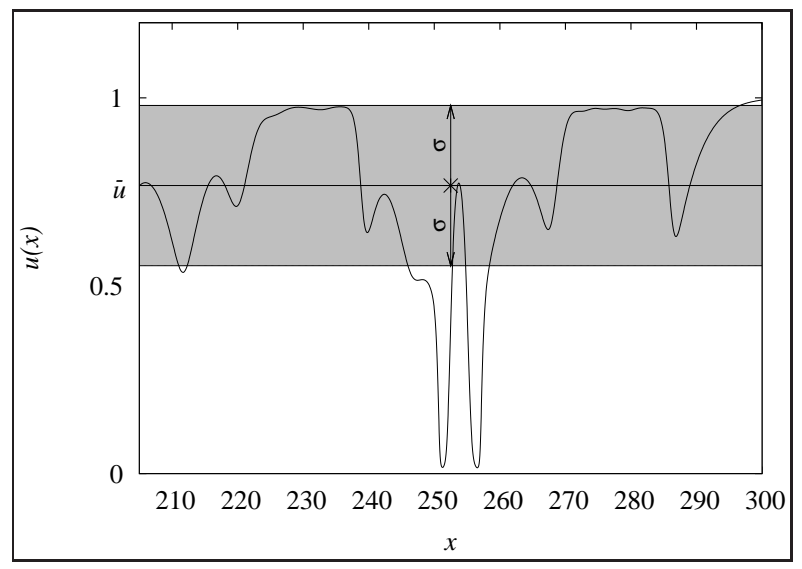

(c) $\mathrm{t}=2335$

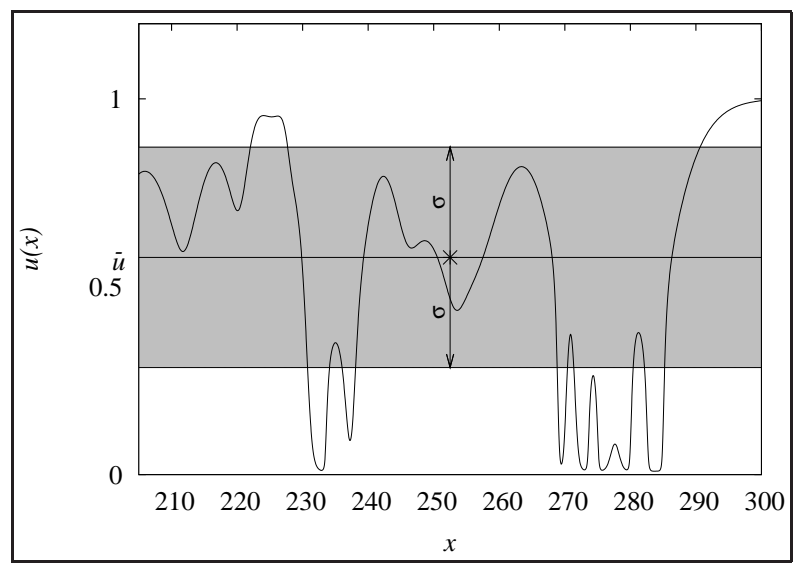

(b) $t=2325$

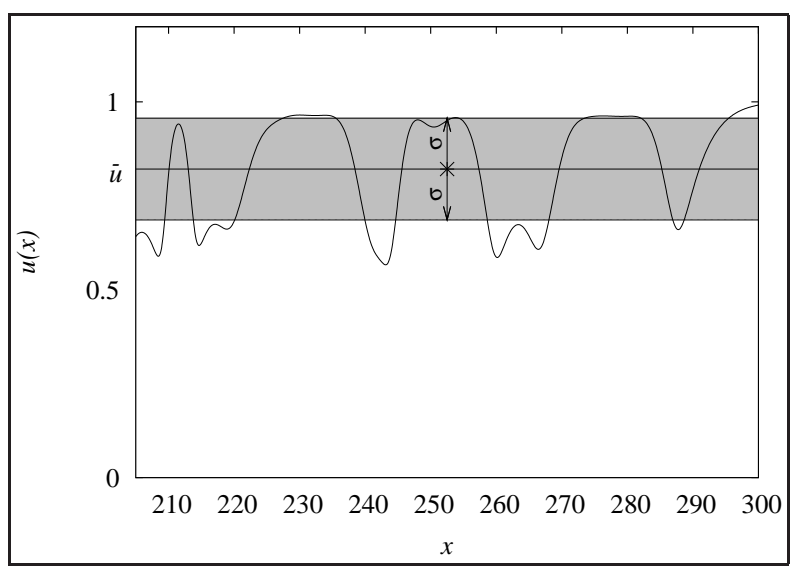

(d) $\mathrm{t}=2360$

Figure 5: A collapse of the prey population is characterised by a drop of the spatial average prey level $\bar{u}$ and an increase of the standard deviation $\sigma$. Parameters given in (3.1).

The average prey level $\bar{u}(t)$ is computed for each time step over the spatial area where the 
predator is above the critical value $v_{\text {min }}$, thus, $\bar{u}(t)$ is a spatial average over the domain

$$
S_{V}:=\left\{x \in \mathbb{R} \mid v(t, x)>v_{\min }\right\} .
$$

This cuts off the right and the left end of the space which have not been invaded yet by the predator and where the prey population is close to its carrying capacity. Low values of this average provide an indicator for the possibility that both populations go extinct on a larger spatial scale. Because of the strong heterogeneity of the pattern also the standard deviation $\sigma(t)$ from this average is taken into account; the interval which contains all prey levels which differ at most one standard deviation from the spatial average is indicated by a grey area in the plots. With these statistical indicators it can be shown quantitatively that the collapses are local, see Figure 5 for a "typical collapse" and Figure 10b for the temporal development of $\bar{u}(t)$ and $\sigma(t)$ : The average population level is always clearly above 0.5 , see Figure 10b. Also, prey levels at places where a collapse takes place differ much more than one standard deviation from the spatial average which shows that these events in a statistical sense can be considered as "exceptions" or in other words: as local events. However, despite being global statistical quantities, the spatial average $\bar{u}(t)$ and the standard deviation $\sigma(t)$ from this average provide indicators for the local collapses: When collapses occur, the average prey level decreases and the standard deviation increases.

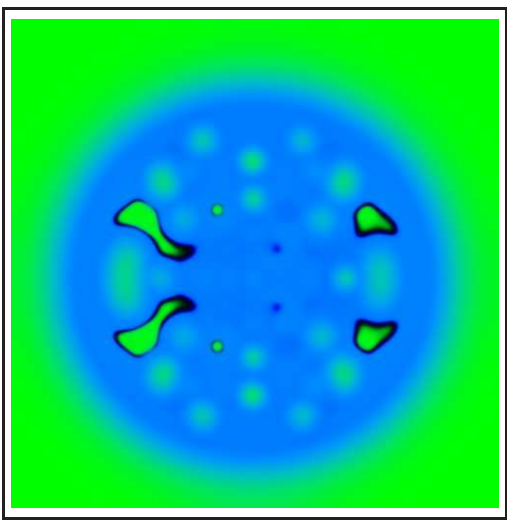

(a) $t=3570$

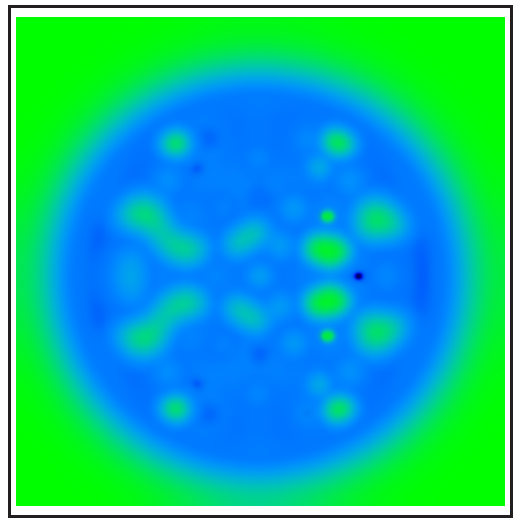

(b) $t=3700$

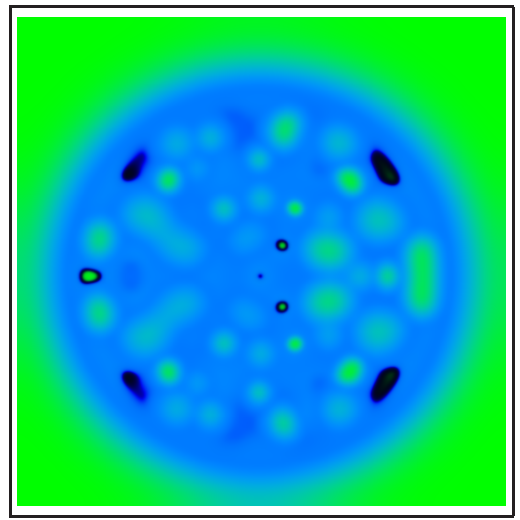

(c) $\mathrm{t}=3785$

Figure 6: For a higher value of $\mu_{V}$ (closer to the Hopf bifurcation $H_{2}$, see Figure 1), a "moonscape" pattern appears: In most of the spatial area the prey level is at a relatively high value (blue "moon"). Black and green spots ("mountains" and "craters") mark areas where the prey level is either nearly at its carrying capacity or close to extinction. Opposed to the first simulation, see Figure 4, local collapses do not "move" through the prey distribution along complex tracks—collapses rather occur in some distance from each other.

Different patterns are obtained for the same initial condition as above (see Figure 3) and the parameter set

$$
\rho=2.0, \mu_{V}=0.996, h=0.03125, d=0.05,
$$


where $\mu_{V}$ is close to the Hopf threshold $\mathrm{H}_{2}$, see Figure 1: A "moonscape" pattern is formed (see Figures 6a-6c). The population levels are nearly constant on the expanding "lunar disk", however, black "craters" appear which indicate local collapses. As to be expected from the local dynamics (see Figure 2), the prey immediately regrows to its carrying capacity (green "mountains"); however, the predator recovers only very slowly. Opposed to the first simulation, collapses do not "travel" along a complex cellular patterns but rather appear as discrete black spots.

\section{Noisy spatio-temporal dynamics}

The model is now extended by density-dependent Gaussian noise which provides a simple model for fluctuations of the populations due to stochastic variability $[1,2,20,21]$. For a recent review on structures which can emerge due to noise, see Sagués et al. [19], also the monograph [4] introduces to effects of noise in spatially extended systems; Lindner et al. [9] give a review on noise in excitable systems. The noise which we will use in the following is "white" in time but "coloured" in space, i.e. the Gaussian random numbers are uncorrelated as time series but in the spatial domain they form a Gaussian random field with a certain correlation. The Gaussian random fields were generated with the algorithm described by Lang [8]. One reason for using spatially correlated noise is the fact that the solutions of stochastic partial differential equations are not guaranteed to be continuous if spatially uncorrelated noise is used, see the monographs $[3,24]$ for more details. Also from the biological point of view, the use of spatially correlated noise makes sense because many types of fluctuations-like, for example, nutrient and temperature differences-which locally increase or decrease the quality of a certain place are indeed correlated.

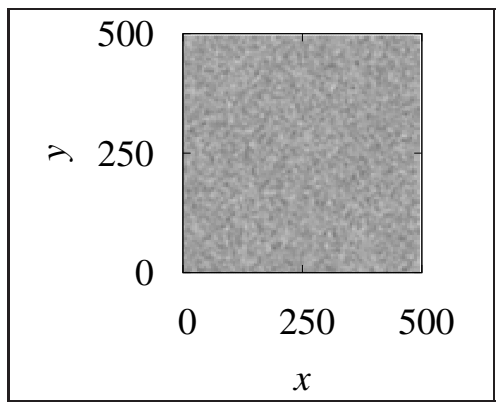

(a) white noise

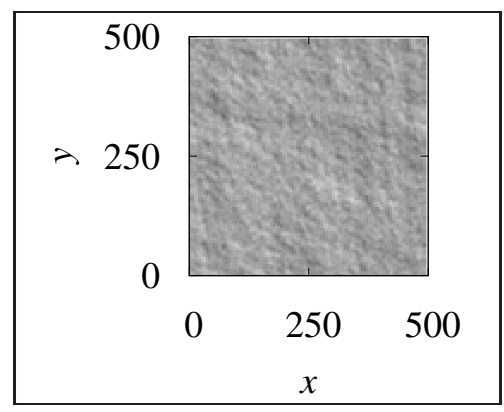

(b) pink noise

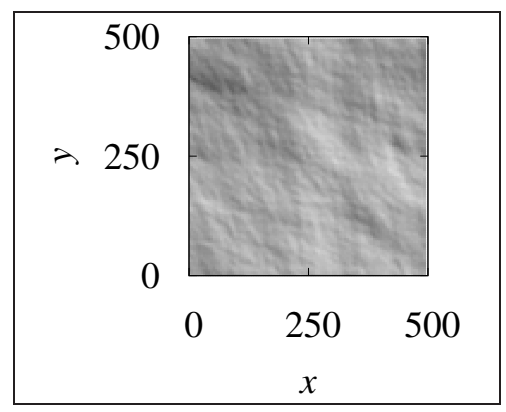

(c) red noise

Figure 7: Samples of noise with different spatial correlations: white (uncorrelated), pink and red noise.

Colours of noise are characterised by their power distribution in the frequency spectrum. Different decays proportional to the frequency $f$ are distinguished. Noise with spectral density per unit of bandwidth proportional to $1 / f$ is denoted "pink", proportionality to $1 / f^{2}$ is characteristic for "red" (sometimes also referred to as "brown") noise. Uncorrelated noise has a flat spectrum 
and is called "white". Three samples of Gaussian random fields with different noise colours are shown in Figure 7.

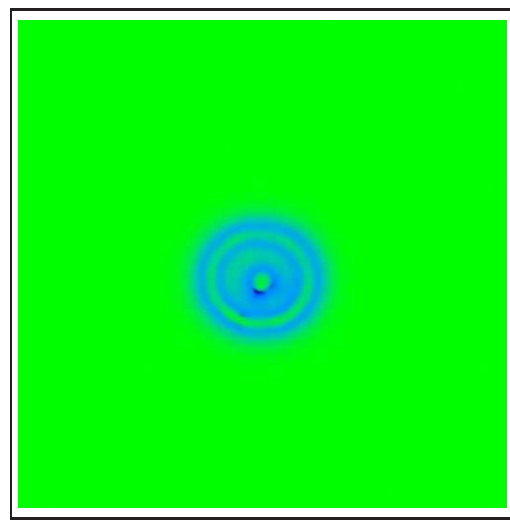

(a) $t=580$

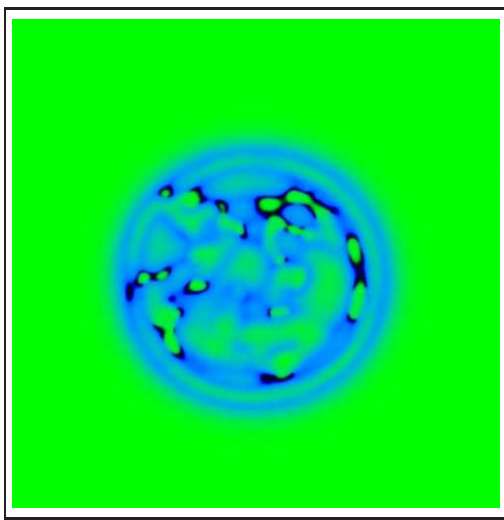

(d) $t=1240$

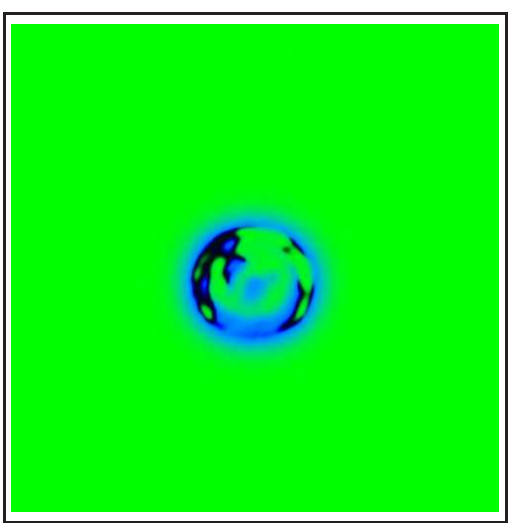

(b) $t=640$

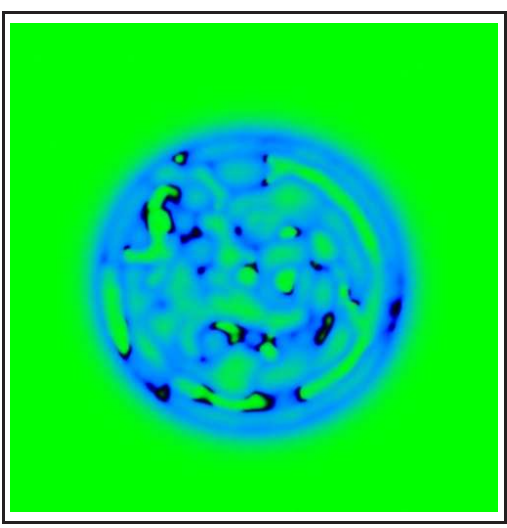

(e) $t=1425$

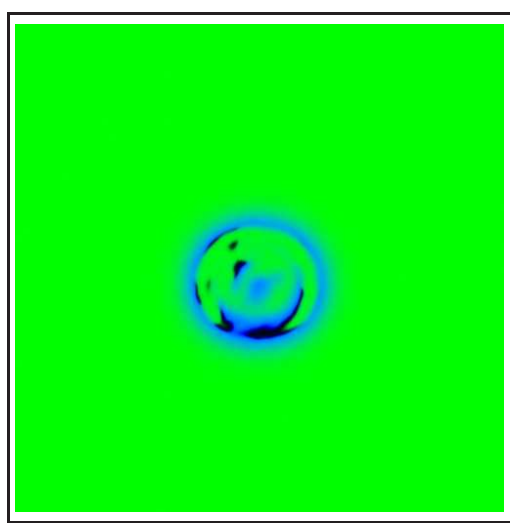

(c) $t=645$

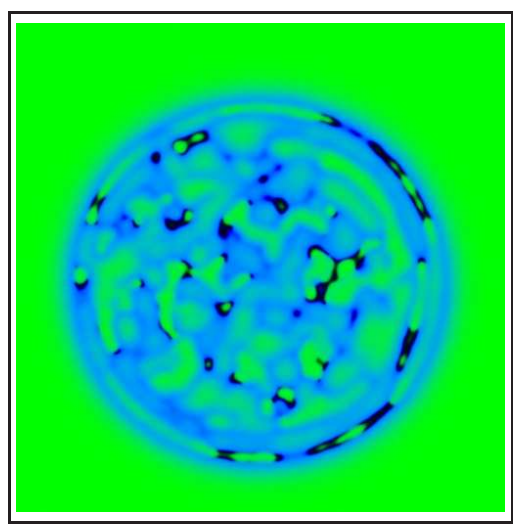

(f) $\mathrm{t}=1700$

Figure 8: Simulation of (2.1) for the parameter set (3.1) and density-dependent "pink" noise. A comparison with the corresponding deterministic solution, see Figure 4, shows that the stochastic system produces similar patterns if no collapses take place, see (a) and compare with Figure 4a. However, the symmetrical distribution of collapses which was observed in the deterministic solutions (see Figure 4) is replaced by an irregular pattern of collapses which however still move along the light blue areas between the green "cells".

First we present solutions with "pink" noise. Again, the parameter set (3.1) was chosen in order to compare the results with the deterministic model. At a low noise intensity $\omega=0.01$ the noise is hardly visible in the results-because the typical granular appearance of the solutions which are usually connected with noise is missing, see Figure 8.

However, already after a few hundred time steps the symmetry of the deterministic solutions is destroyed by local collapses whose locations are distributed irregularly opposed to the deter- 


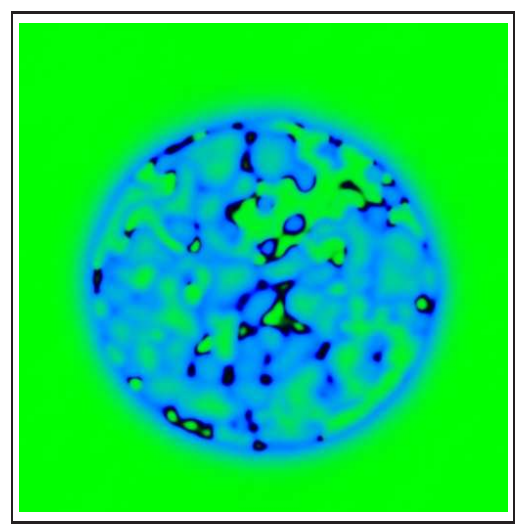

(a) $\mathrm{t}=1575$

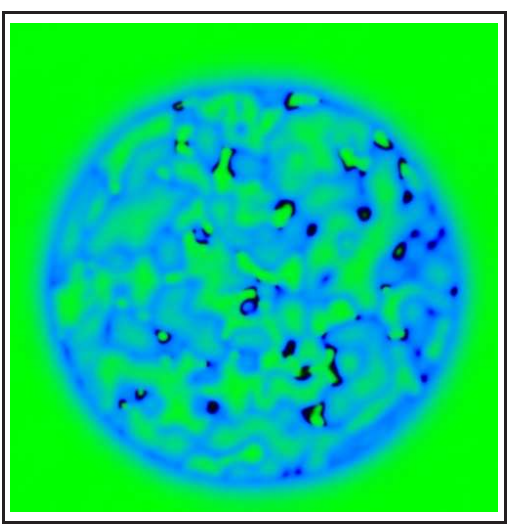

(b) $\mathrm{t}=1850$

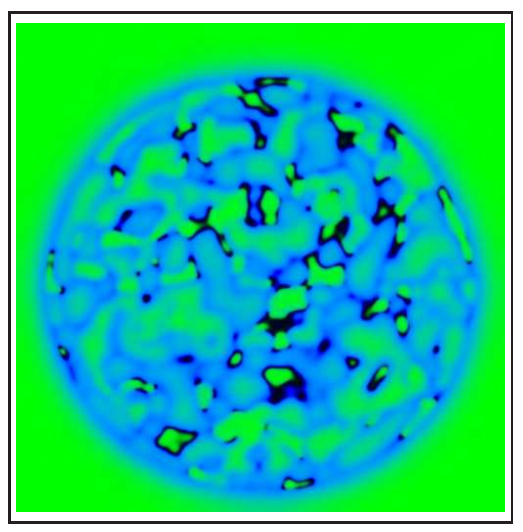

(c) $t=1930$

Figure 9: Simulation of (2.1) for the parameter set (3.1) and density-dependent "red" noise. It seems that inside the cellular structure which was also formed in the deterministic simulation, see Figure 4, the collapses are spatially more extended. This could be explained by the stronger correlation of the Gaussian random field. Generally, the solution is qualitatively similar to the corresponding simulation with "pink" noise, see Figure 8.

ministic model where some symmetries could still be received. Interestingly, also the stochastic pattern indeed looks nearly symmetric when the system is not under the influence of "inverse excitability", i.e. if there are no collapses, see Figure 8a and compare with Figure 4a. The typical enhancement of the diffusive movement compared to the deterministic system, cf. for example [4], is also observed in this model.

Driving (2.1) with "red" noise produces similar dynamics. The stronger spatial correlation seems to increase the spatial extension of the collapses, see Figure 9. Potentially this might change the results which were obtained from the deterministic model, i.e. under the influence of strongly correlated noise the tendency of the system to exhibit local collapses might be an extinction risk. A more systematic study of the extension of collapses depending on the spatial correlation of the noise is postponed to a subsequent paper.

\section{Conclusions}

A new aspect of the spatio-temporal behaviour of the Truscott-Brindley model was discovered: Close to but below the Hopf threshold $H_{1}$, the solutions are characterised by the well-known phenomenon of excitability. In the spatio-temporal context, this means that a small local perturbation of the stationary prey level may lead to "excitation", i.e. a rapid growth of the prey population which is followed by relaxation, i.e. a decrease to the stationary resting state. This type of solution was, for example, related to phytoplankton blooms [22]. Our simulations without noise show that close to but below the Hopf threshold $\mathrm{H}_{2}$ when the dynamics are characterised by relaxation oscillations, the spatio-temporal dynamics looks exactly the opposite way: The prey population is 
at a high level close to its carrying capacity; however, small perturbations lead to collapses after which the prey regrows rapidly — we find some kind of "inverse excitability". It becomes clear that the spatio-temporal dynamics close to both Hopf thresholds is "mirrored"- thus, a rather subtle symmetry of the model was revealed. Further work will investigate if this is a general feature of excitable systems or which additional conditions have to be imposed.

Driving with temporally white spatially coloured noise blurs artificial structures of the deterministic solutions which is a well-known effect of noise. However, the spatial correlation of stochastic forcing is especially interesting in our case of "inverse excitability": The correlation of the stochastic perturbations tend to increase the spatial extension of collapses. This has to be determined more systematically in a further study, however, already this qualitative result has a clear ecological implication: Even though in the deterministic model the population levels are high, the tendency of showing local collapses might be catastrophically amplified by spatially correlated environmental fluctuations leading to extinction of major parts of the populations on a larger spatial scale.

Finally, there is a simple but nice relation to the famous paradox of enrichment, see [17]: It was mentioned above (see Section 3.) that by choosing a low value of $h$, the system can be interpreted as "enriched" because the carrying capacity is high compared to the half saturation constant $H$ of the predator. Indeed, sharp oscillations develop which, in the context of the local model, was taken as a hint that populations might be destabilised and even go extinct- despite the increased carrying capacity which should intuitively lead to stabilisation. However, in this respect, the simulations which are presented here show that in most of the spatial area both populations maintain relatively high levels far away from extinction! This suggests an especially simple resolution of the paradox of enrichment which only depends on two ingredients: the dynamics characterised by slow-fast cycles and the spatial extension of the system. Heuristically the transition of an oscillatory local model to the corresponding spatio-temporal model can be made by assuming that at each point in space oscillators are coupled to their neighbourhood by diffusion, see for example [7] for an introduction to this idea. For most of the time, the prey population is at a high level because this is the "slow" part of its slow-fast cycle; collapses are nearly instantaneously followed by rapid regrowth to the carrying capacity. This reasoning consistently leads to the heuristic assumption that in most of the spatial area the prey level must be at a high level-the only exception is of course the case when the coupling between the oscillators is so strong that the prey level starts to oscillate homogeneously. In this sense, our results are closely related with [14]. However, Petrovskii et al. [14] were mainly interested in "enrichment" as a process, i.e. opposed to our approach, they actually considered an increase of the carrying capacity whereas we already start in a situation with a supossedly high value of $K$. Also, Petrovskii et al. [14] used the RosenzweigMacArthur model [18] with Holling type II functional response of the predator (for a summary of the main results of [14] and a wealth of spatio-temporal patterns found with the RosenzweigMacArthur model, see Malchow et al. [11, chapters $10 \& 11]$ ). They present spatio-temporal chaos as a possible resolution of the paradox of enrichment in spatially extended systems. Our results suggest that in fact even the slow-fast dynamics itself is enough to ensure the survival of populations in spatially extended systems. To understand this we compare the average prey level 


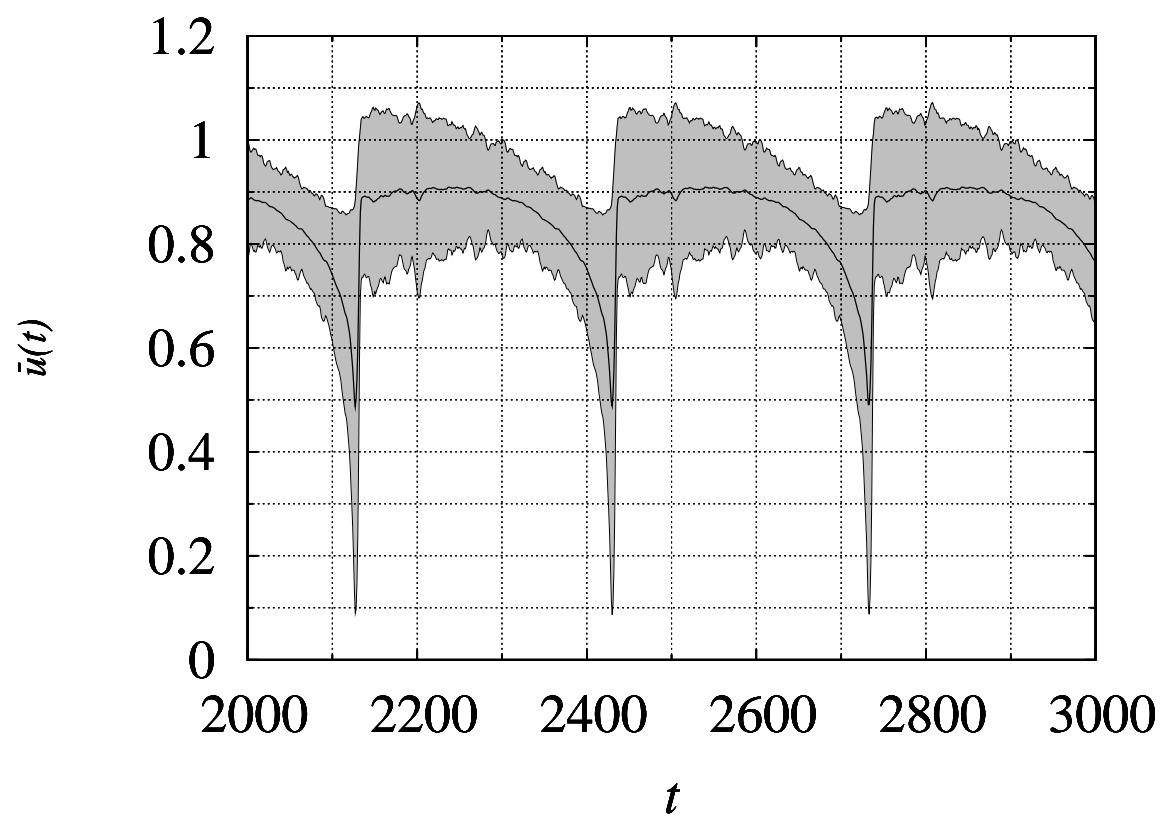

(a) average of 500 solutions of (2.1) without diffusion

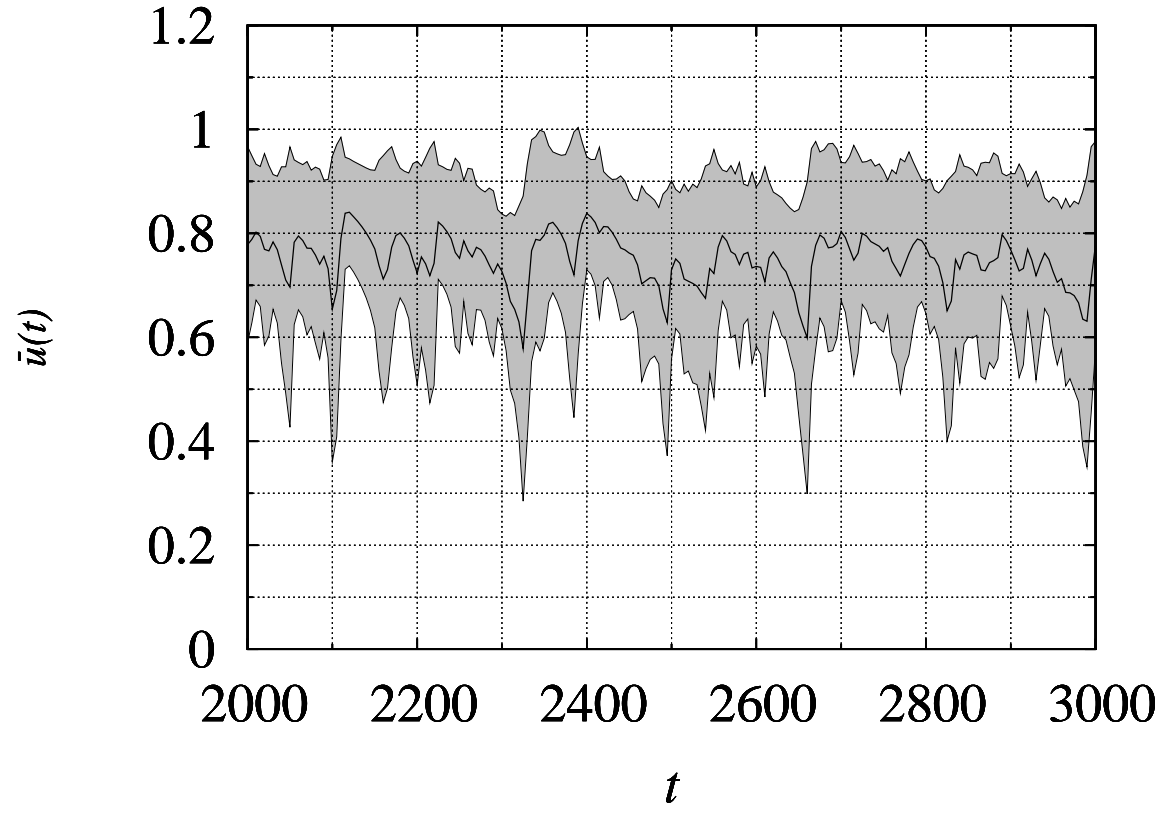

(b) spatially averaged prey level

Figure 10: Comparison of the average prey level $\bar{u}(t)$ for 500 solutions $u_{i}(t)$ of (2.1) with randomly chosen, uniformly distributed initial conditions with the spatially averaged prey level of the model with diffusion in one-dimensional space. The standard deviation from the average is shown by the grey area.

of a set $u_{i}(t)$ of 500 solutions of the deterministic ODE system without diffusive movement of the species. The initial conditions are randomly chosen and uniformly distributed - thus every 
solution $u_{i}(t)$ reaches the same limit cycle, however, two different solutions $u_{i}(t)$ and $u_{j}(t), i \neq j$ usually differ by a phase shift. The average prey level $\bar{u}(t)$ of these 500 solutions and the standard deviation $\sigma(t)$ are computed and plotted for the time interval $t \in[2000,3000]$, see Figure 10a.

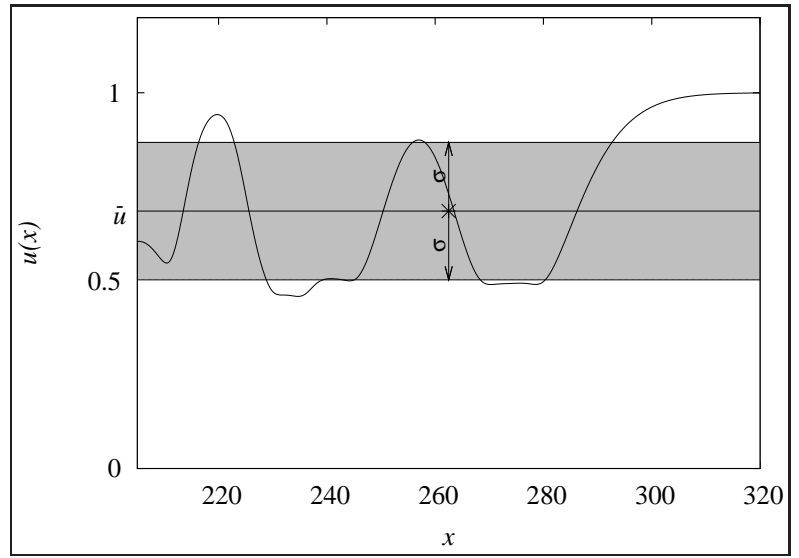

(a) $\mathrm{t}=4600$

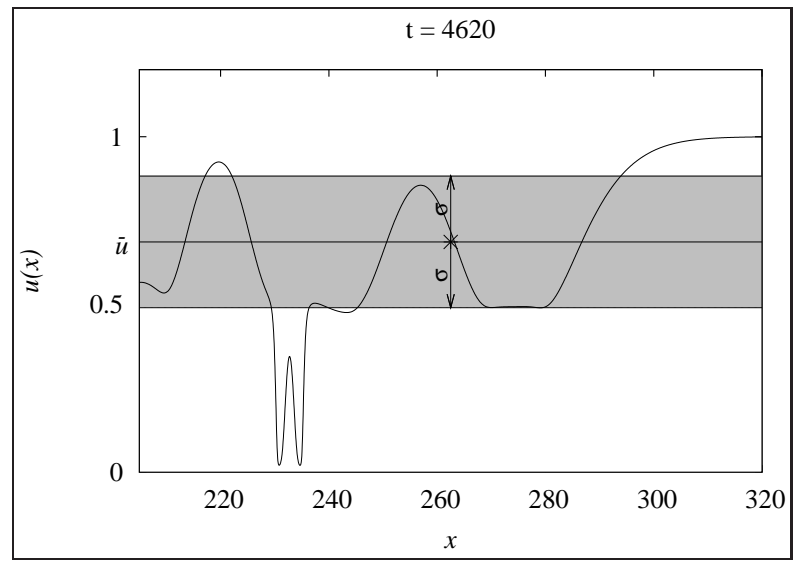

(c) $\mathrm{t}=4620$

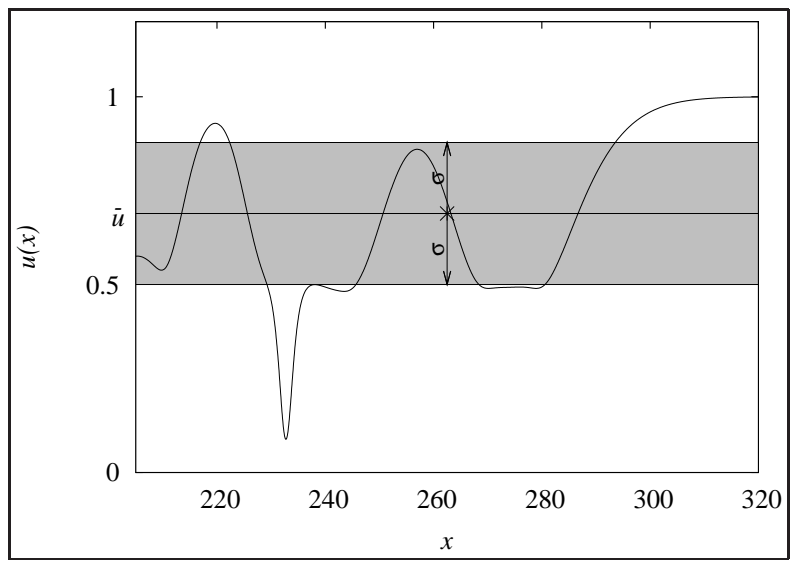

(b) $\mathrm{t}=4615$

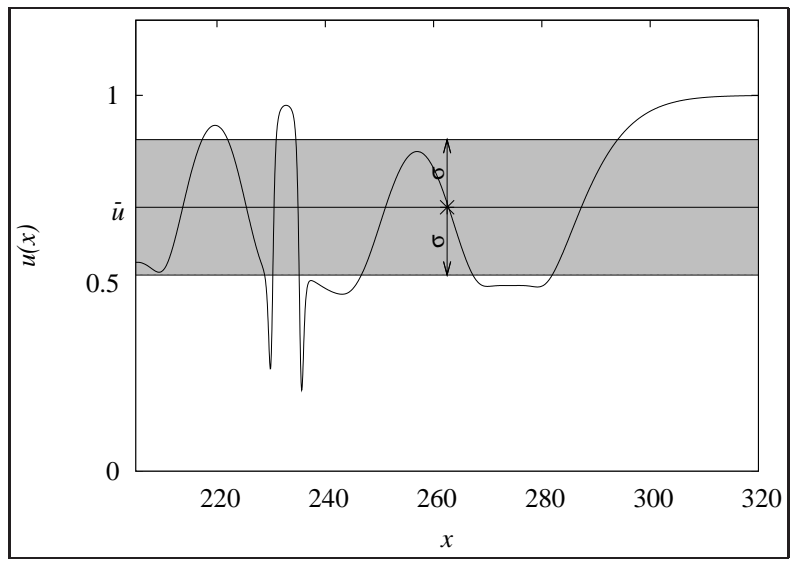

(d) $t=4625$

Figure 11: Although in the local model for the parameters (3.2) the oscillations are sharper than for the parameter set (3.1), the behaviour of the spatial model 2.1 is in fact more stable. This can be explained by the slow-fast dynamics: The series of pictures shows that the prey distribution seems to be stationary except for a small region at $x \approx 230$ where the prey level collapses and quickly regrows to its carrying capacity.

By simple considerations one finds that $\bar{u}(t)$ and $\sigma(t)$ have the same period $T$ as all $u_{i}(t)$. Also, $\bar{u}(t)$ has a smaller maximum $\bar{u}_{\max }$ and a larger minimum $\bar{u}_{\min }$ than the single solutions $u_{i}(t)$, i.e. $u_{\min } \leq \bar{u}_{\min }<\bar{u}_{\max } \leq u_{\max }$. This argument already shows that-even if there is no coupling by diffusion - the average population level $\bar{u}(t)$ of solutions $u_{i}(t)$ which are shifted out of phase is far away from extinction (especially in the case of relaxation oscillations as the prey is at a high level in the "slow" phase of the dynamics and only stays at a low level for a short instant in the 
"fast" phase). The fact that the solutions $u_{i}(t)$ have to be out of phase to considerably "quench" the amplitude of $\bar{u}(t)$ probably convinced Petrovskii et al. [14] that the spatial pattern shall be characterised by spatio-temporal chaos.

However, simulations of the parameter set (3.2) show that if the oscillations in the local model are especially sharp, the spatial system shows quasi-stationary population distributions which hardly change-except for small "hot spots" where collapses occur. The sharpness of the oscillations is in fact the main reason that the system stabilises quickly after the collapse, see Figure 11 and compare Figure 12 with Figure 10b. Thus, the paradox of enrichment seems to be resolved quite naturally in systems whose dynamics is characterised by relaxation oscillations.

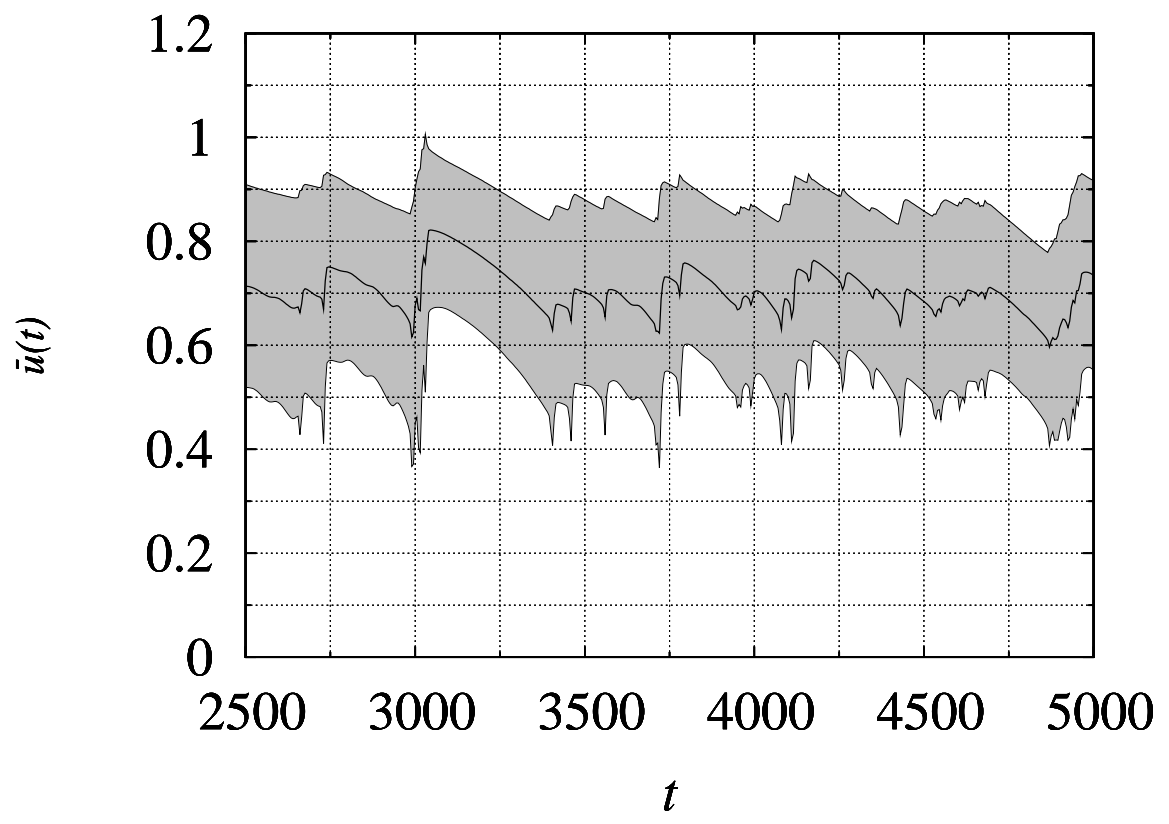

Figure 12: Spatially averaged prey level for (2.1) in one-dimensional space. The standard deviation $\sigma(t)$ from the spatial average $\bar{u}(t)$ is shown by the grey area. Although the oscillations in the local model are even sharper for the parameter set (3.2) than for (3.1), the spatial solution is not influenced as strong by collapses_-both spatial average $\bar{u}(t)$ and standard deviation $\sigma(t)$ show less variability, cf. Figure 10b.

\section{Acknowledgements}

Most of the research for this article was accomplished during a two-month stay at the Ben Gurion University in Beer Sheva, Israel. IS thanks for the friendly hospitality of Ehud Meron and his group and acknowledges the support of the DAAD for this very fruitful visit. The comments of two anonymous reviewers which improved the manuscript a lot are also gratefully acknowledged. 


\section{References}

[1] L. J. S. Allen. An introduction to stochastic processes with applications to biology. Pearson Education, Upper Saddle River NJ, 2003.

[2] V. S. Anishenko, V. V. Astakov, A. B. Neiman, T. Vadivasova, L. Schimansky-Geier. Nonlinear dynamics of chaotic and stochastic systems. Tutorial and modern developments. Springer Series in Synergetics. Springer, Berlin, 2003.

[3] P.-L. Chow. Stochastic Partial Differential Equations. Applied Mathematics and Nonlinear Science. Chapman \& Hall / CRC, Boca Raton, 2007.

[4] J. García-Ojalvo, J. M. Sancho. Noise in spatially extended systems. Institute for Nonlinear Science. Springer, New York, 1999.

[5] C. S. Holling. Some characteristics of simple types of predation and parasitism. The Canadian Entomologist, 91 (1959), No. 7, 385-398.

[6] A. Y. Kolesov, Y. S. Kolesov. Relaxation Oscillations in Mathematical Models of Ecology, vol. 199 of Proceedings of the Steklov Institute of Mathematics. American Mathematical Society, Providence, Rhode Island, USA, 1995.

[7] Y. Kuramoto. Chemical oscillations, waves, and turbulence, vol. 19 of Springer Series in Synergetics. Springer, Berlin, 1984.

[8] A. Lang. Simulation of Stochastic Partial Differential Equations and Stochastic Active Contours. Dissertation, Universität Mannheim, Institut für Mathematik, Lehrstuhl für Mathematik V (2007).

[9] B. Lindner, J. Garcia-Ojalvo, A. Neiman, L. Schimansky-Geier. Effects of noise in excitable systems. Physics Reports, 392 (2004), 321-424.

[10] D. Ludwig, D. D. Jones, C. S. Holling. Qualitative analysis of insect outbreak systems: the spruce budworm and forest. Journal of Animal Ecology, 47 (1978), 315-332.

[11] H. Malchow, S. V. Petrovskii, E. Venturino. Spatiotemporal Patterns in Ecology and Epidemiology - Theory, Models, and Simulation. Mathematical and Computational Biology Series. Chapman \& Hall / CRC, Boca Raton, 2008.

[12] E. F. Mishchenko. Asymptotic calculation of periodic solutions of differential equations with small parameters in front of the derivatives. Izvestiya Akademii Nauk SSSR, 21 (1957), 627-654.

[13] E. F. Mishchenko, L. S. Pontryagin. Periodicheskie resheniya sistem differentsialnykh uravnenii, blizkie k razryvnym. Doklady Akademii Nauk SSSR, 102 (1955), 889-891. 
[14] S. V. Petrovskii, B.-L. Li, H. Malchow. Transition to spatiotemporal chaos can resolve the paradox of enrichment. Ecological Complexity, 1 (2004), No. 1, 37-47.

[15] L. S. Pontryagin. Asymptotic behaviour of solutions of systems of differential equations with a small parameter in front of the highest order derivatives. Izvestiya Akademii Nauk SSSR, 21 (1957), 605-626.

[16] S. Rinaldi, M. Scheffer. Geometric analysis of ecological models with slow and fast processes. Ecosystems, 3 (2000), 507-521.

[17] M. L. Rosenzweig. Paradox of enrichment: Destabilization of exploitation ecosystems in ecological time. Science, 171 (1971), 385-387.

[18] M. L. Rosenzweig, R. H. MacArthur. Graphical representation and stability conditions of predator-prey interactions. The American Naturalist, 97 (1963), 209-223.

[19] F. Sagués, J. M. Sancho, J. García-Ojalvo. Spatiotemporal order out of noise. Reviews of Modern Physics, 79 (2007), 829-882.

[20] B. Spagnolo, M. Cirone, A. La Barbera, F. de Pasquale. Noise-induced effects in population dynamics. Journal of Physics: Condensed Matter, 14 (2002), 2247-2255.

[21] B. Spagnolo, D. Valenti, A. Fiasconaro. Noise in ecosystems: a short review. Mathematical Biosciences and Engineering, 1 (2004), No. 1, 185-211.

[22] J. Truscott, J. Brindley. Ocean plankton populations as excitable media. Bulletin of Mathematical Biology, 56 (1994), 981-998.

[23] B. van der Pol. On "relaxation-oscillations". The London, Edinburgh, and Dublin Philosophical Magazine and Journal of Science Ser.7, 2 (1926), 978-992.

[24] J. B. Walsh. An introduction to stochastic partial differential equations. In R. Carmona, H. Kesten, J. B. Walsh (Eds.), École d'été de probabilités de Saint-Flour XIV - 1984, vol. 1180 of Lecture Notes in Mathematics. Springer, Berlin, 1986. 